Published in final edited form as:

Sleep Breath. 2020 March ; 24(1): 159-166. doi:10.1007/s11325-019-01845-z.

\title{
Do symptoms of sleepiness and insomnia in US veterans with obstructive sleep apnea vary by age?
}

\author{
C. Agudelo ${ }^{1,2}$, A.R. Ramos ${ }^{1}$, N.J. Williams ${ }^{3}$, D.M. Wallace ${ }^{1,2}$ \\ ${ }^{1}$ Department of Neurology, Sleep Medicine Division, University of Miami Miller School of \\ Medicine, Miami, FL, USA \\ ${ }^{2}$ Neurology Service, Bruce W. Carter Department of Veterans Affairs Medical Center, 1201 NW \\ 16th Street, Miami, FL 33125, USA \\ ${ }^{3}$ Department of Population Health, New York Langone Health, Center for Healthful Behavior \\ Change, New York, NY, USA
}

\begin{abstract}
Introduction-The influence of aging on the clinical presentation of obstructive sleep apnea (OSA) is not well characterized in US veterans. Our aims were to (1) examine age and established predictors of sleepiness and insomnia symptoms in veterans with OSA and (2) determine if the relationship between predictors of the Epworth sleepiness scale (ESS) and insomnia severity index (ISI) depended on age.
\end{abstract}

\begin{abstract}
Methods-We performed a retrospective analysis of veterans diagnosed with OSA at the Miami VA in 2014. On polysomnography (PSG) night, questionnaires were completed querying sociodemographics, insomnia (ISI), sleepiness (ESS), and self-reported sleep duration. Regression modeling was performed to explore association of variables with (1) ESS and (2) ISI. Analyses were performed in two steps: (1) variables were tested for main effects and (2) product of age and each variable found to have an association at a significance level of $p<0.10$ with primary outcome were entered separately to test for interaction.
\end{abstract}

Results-The sample consisted of 483 veterans (93\% male, age $52 \pm 13$ years, $41 \%$ black, $34 \%$ Hispanic). Having a regular bed partner, higher weighted medical comorbidities, chronic pain diagnosis, and shorter sleep duration were associated with ESS. Age did not moderate the relationship between these variables and ESS. Younger age, Hispanic ethnicity, higher educational level, shorter sleep duration, mood, and pain diagnoses were each associated with the ISI. Furthermore, an age-sleep duration interaction term was associated with the ISI $(b=-0.03 ; p=$

\footnotetext{
D.M. Wallace dwallace@med.miami.edu.

Compliance with ethical standards

Conflict of interest The authors declare that they have no conflict of interest.

Ethical approval All procedures performed in studies involving human participants were in accordance with the ethical standards of the institutional and/or national research committee and with the 1964 Helsinki declaration and its later amendments or comparable ethical standards.

Informed consent As this study involved retrospective analysis of existing clinical data, informed consent was not obtained from participants.

Publisher's note Springer Nature remains neutral with regard to jurisdictional claims in published maps and institutional affiliations.
} 
0.005). For all participants, there was an inverse relationship between sleep duration and ISI. However, for any sleep duration, older veterans reported lower levels of insomnia than younger veterans.

Discussion-Older veterans with OSA may report lower ISI scores. Alternative assessment methods for comorbid insomnia among older individuals with OSA may be needed.

\section{Keywords}

Veterans; Insomnia; Obstructive sleep apnea; Sleepiness; Age

\section{Introduction}

Obstructive sleep apnea (OSA) is of great public health concern due to its increasing prevalence with aging and associated mental and medical comorbidities [1]. The prevalence of OSA among those over the age of 65 is 2-3 fold higher than that of middle-aged adults, partially mediated through greater upper airway collapsibility, reduced arousal threshold, and reduced lung volumes [2]. The estimated prevalence of OSA among the US general population ranges between 9 and 38\% [1]. Importantly, among the US military, the rates of medical encounters have grown by $372 \%$ for insomnia and $517 \%$ for OSA over a 10 -year period (2004-2015) with the greatest increases seen among older and black individuals [3]. Given this rising burden of treatable sleep complaints among military service members, understanding the various clinical presentations of OSA is particularly timely.

Aging can influence the evaluation of OSA including reports of subjective daytime sleepiness and comorbid insomnia symptoms [4]. For example, the Epworth sleepiness scale (ESS), one of the most common screening instruments for assessing sleepiness, may be limited among older individuals as they may not perform all of the queried daytime activities [5]. Similarly, insomnia symptoms may be under-reported in older individuals with OSA. In population-based studies, among those with moderate to severe OSA, older individuals were found to report fewer insomnia symptoms [6]. Furthermore, as the prevalence of sleeping alone increases with age, the absence of a bed partner can complicate further OSA risk stratification. Thus, many older individuals may be less likely to be diagnosed with OSA partially due to inadequate assessment of common OSA symptoms.

The association of comorbid conditions and racial/ ethnic background in the report of sleepiness and insomnia in OSA is less well characterized in military veterans. Among service members completing diagnostic polysomnography (PSG), Mysliwiec et al. reported that $38 \%$ of individuals had insomnia comorbid with OSA [7]. In addition, post-traumatic stress disorder (PTSD) and depression were identified as risk factors for insomnia comorbid with OSA [7]. However, predictors of comorbid insomnia in military samples with OSA have not been comprehensively described. Furthermore, an individual's cultural background may be important in the evaluation of normative values of sleepiness and insomnia symptoms. For example, blacks may interpret items on the ESS differentially than whites [8]. In addition, responses among minority individuals may vary depending on how insomnia symptoms are assessed [9]. Despite that military service members have higher prevalence of mental health comorbidities (e.g., PTSD) than civilians and a high pro-portion 
of individuals from minority background, there are little data on whether generational effects exist between comorbidities or racial/ethnic background and reports of sleepiness or insomnia in OSA [3, 10]. More comprehensive descriptions of OSA clinical presentations among culturally diverse samples are warranted to adequately identify at-risk individuals. Therefore, the aims of this study were to examine (1) age and established medical and psychosocial predictors of daytime sleepiness and insomnia symptoms in a culturally diverse sample of US veterans with OSA and (2) determine if the relationship between these predictors and the Epworth sleepiness scale and the insomnia severity index depended on age.

\section{Methods}

\section{Study sample}

Participants were 483 veterans newly diagnosed with OSA over a 1-year period (JanuaryDecember 2014) at the Miami Veterans 'Administration Healthcare System (VAHCS) sleep center. Inclusion criteria included (1) new OSA diagnosis (ICSD-3 criteria) at the Miami VAHCS over 1-year period and (2) completion of study questionnaires. Exclusion criteria were history of prior diagnosis or treatment of OSA and unstable medical/psychiatric comorbidity.

\section{Procedures}

Individuals included in this retrospective analysis completed PSG at the Miami VAHCS sleep center between January and December 2014. Individuals either completed in-lab overnight PSG ( $n=367$; REMLOGIC) or unattended home PSG ( $n=116$; Embletta Gold type III recorder; Embla, Thorton, CO). We conducted diagnostic in-lab and portable PSGs in accordance with standards established by AASM [11, 12]. Scoring was performed manually by a certified sleep technologist, using 30-s epochs and standardized scoring techniques [13]. Hypopnea scoring followed the recommended definition ( $\geq 30 \%$ reduction in nasal pressure relative to baseline associated with $\geq 4 \%$ oxygen desaturation). Prior to all PSGs, individuals are asked to complete questionnaires querying sociodemographic details (age, gender, race, ethnicity, marital status, education, work schedule), medical/psychiatric comorbidities, sleep symptoms, and habitual sleep duration (detailed below). The Miami VAHCS Institutional Review Board approved the protocol.

\section{Assessments}

\section{Medical record data}

Polysomnography: Details concerning completed PSG were extracted from the electronic medical record. These included the diagnostic PSG study type, apnea-hypopnea index (AHI), and oxygen desaturation nadir. Height and weight measured on the night of PSG were obtained to calculate the body mass index (BMI).

Medical diagnoses data-Medical history and comorbidities were obtained from questionnaires and were verified by review of electronic medical records. Individuals were categorized as having a chronic medical disorder if they self-reported the condition and if they were prescribed medical treatment for this disorder at the Miami VAHCS. Weighted 
medical comorbidities were calculated using the Charlson comorbidity index (CCI) [14]. A diagnosis of chronic pain was assigned to (1) individuals prescribed medication for pain complaints (e.g., NSAIDs, opioids, muscle relaxants) for $\geq 6$ months preceding diagnostic PSG or (2) individuals attending the Miami VAHCS pain clinic in the 12 months preceding diagnostic PSG.

Psychiatric diagnoses data-Similar to the medical disorders, individuals were categorized as having depressed mood or PTSD if they self-reported either condition and were diagnosed or treated for a mood disorder (major depressive disorder and bipolar disease) or PTSD, respectively, by the psychiatry service at the Miami VAHCS.

Zip code socioeconomic status-Each veteran's residential zip code was used to generate a median annual household income according to 2010 US Census data [15].

Sleep questionnaires-Participants completed the insomnia severity index (ISI), a 7item instrument measuring the individual's perception of his or her insomnia. The total scores range from 0 to 28 with higher scores indicating greater insomnia severity. Its internal consistency, concurrent validity, and sensitivity to clinical improvements are well established [16].

Subjective daytime sleepiness was determined using the Epworth sleepiness scale (ESS), with 8 items rated on a scale of $0-3$ and higher scores indicating a greater propensity to fall asleep in different situations [17].

Habitual sleep times during the month prior to the diagnostic PSG were determined using the first four items of the Pittsburgh sleep quality index (PSQI) [18]. These items assess typical bed time, sleep onset latency, out of bed time, and total sleep time (TST). Time in bed (TIB) was calculated using the reported bed time and out of bed time.

\section{Statistical analyses}

Preliminary data analysis included descriptive statistics and assessing for normality of distribution. Data are reported as means and SDs for continuous variables and frequencies (\%) for categorical variables.

Separate linear regression modeling was used to explore the association of predictor variables with (1) the Epworth sleepiness scale and (2) the insomnia severity index. Variables selected to predict daytime sleepiness and insomnia symptoms were chosen based on previous research (age, gender, race, ethnicity, BMI, socioeconomic status, medical/ psychiatric comorbidities, AHI, and habitual sleep duration) [19-22]. Educational level and marital status served as individual-level proxies for socioeconomic status (SES) while zip code SES served as a mark for residential SES [23]. Additionally, we adjusted for bed partner status as the presence of a bed partner can influence sleep symptom report [24]. In each model, regression analysis was performed in two steps: (1) all variables were entered simultaneously testing for main effects and (2) the product of age and each variable found to have an association at a significance level of $p<0.10$ with the primary outcome were entered separately to test for interaction. These interaction terms were of primary interest to 
determine if age moderated the relationship of the predictors with daytime sleepiness or insomnia symptoms. For example, do weighted medical comorbidities impact insomnia symptoms differentially in younger versus older veterans? Continuous interaction variables were mean centered. Each interaction term was added separately and was retained in each regression model only if found to be significantly associated with either the ESS or the ISI.

For all models, multicollinearity among predictors was examined by scrutinizing their Pearson correlations, variance inflation factors, eigenvalues, and condition indexes. For all analyses, $p<0.05$ was defined as statistically significant. Statistical analyses were performed with SPSS Statistics 22.0 (SPSS, Chicago, IL). Interaction effects were probed and figures were generated using PROCESS [25].

\section{Results \\ Sample characteristics}

The sample consisted of 483 veterans diagnosed with OSA. Participants were predominantly male (93\%), white (59\%), middle-aged ( $52 \pm 13$ years), and obese (BMI $33 \pm 6 \mathrm{~kg} / \mathrm{m}^{2}$ ).

Thirty percent of participants reported sleeping alone. Forty-one percent of the sample was non-Hispanic black while 34\% of the sample was Hispanic white. Psychiatric comorbidities were prevalent with $56 \%$ and $32 \%$ diagnosed and treated for mood disorder and PTSD, respectively. On average, participants had a severe level of OSA (AHI $36 \pm 28$ events/h of sleep) and reported comorbid insomnia symptoms (ISI of $18 \pm 6$ ) and daytime sleepiness (Epworth score of $12 \pm 6$ ). In addition, short sleep duration was common with mean habitual sleep duration of $5.4 \pm 1.3 \mathrm{~h}$. The rest of the sample characteristics are presented in Table 1 .

\section{Predictors of the Epworth sleepiness scale in OSA}

Among veterans with OSA, we explored the association of several demographic, social, and medical/psychiatric, and polysomnographic variables with the ESS (Table 2). In adjusted analysis, age was not associated with daytime sleepiness. However, main effects were observed for bed partner status, weighted medical comorbidities, chronic pain diagnosis, habitual sleep duration, and the ESS. Specifically, a 1-unit increase in the Charlson index was associated with 0.5 lower ESS score. Similarly, 1-h increment above the mean sleep duration was associated with nearly 0.8 lower ESS score. In contrast, a diagnosis of chronic pain and the presence of a regular bed partner were each associated with a 1.2 and 1.8 higher score on the ESS. We sequentially explored six potential interactions between age and variables associated with ESS at a $p \leq 0.10$ (BMI, bed partner status, sleep duration, Charlson index, pain, and mood diagnoses). Age did not moderate the relationship between any of these variables and daytime sleepiness. Therefore, these terms were removed from the final model.

\section{Predictors of the insomnia severity index in OSA}

Age, Hispanic ethnicity, higher educational level, habitual sleep duration, mood, and pain diagnoses were each significantly associated with the ISI (Table 3). Hispanic ethnicity and a 1-h increase exceeding the mean sleep duration were associated with nearly a 1.4 and 1.65 lower ISI score, respectively. In contrast, educational level beyond high school was 
associated with a 1.15 higher ISI score. Interestingly, a 10-year increment above the mean sample age was associated with a 0.8 lower ISI score. Finally, a mood disorder and chronic pain diagnosis were each associated with a 2.9 and 1.7 higher ISI score, respectively.

Similarly as in the ESS model, each of these six variables was interacted with age and nonsignificant age-interaction terms were removed. As shown in Table 3, an age-sleep duration interaction term was significantly associated with the ISI $(p=0.005)$ and retained in the final model. This age-sleep duration interaction is visualized in Fig. 1. As displayed by the predicted values, there was an inverse relationship between sleep duration and ISI scores for all participants. However, this negative relationship was steeper for older veterans than younger individuals with OSA. For any sleep duration, older veterans reported a lower level of insomnia symptoms than younger veterans. We probed this continuous interaction using the Johnson-Newman technique to determine if this interaction was observed at specific ages [25]; however, the age-sleep duration interaction was significant across the entire age range.

We performed a sensitivity analysis to ensure that symptoms detected by the total ISI originated from nocturnal insomnia symptoms. We repeated the model with the sum of the first three ISI items (nocturnal insomnia symptoms) as the outcome and the same predictors. The main effects and interaction findings were unchanged.

\section{Discussion}

These data explored if the relationship between common predictors of sleepiness and insomnia symptoms found in patients with OSA depends on an individual's age. Specifically, they highlight the importance of psychosocial and medical comorbidity variables in the report of sleepiness and insomnia symptoms. In our racially/ethnically diverse sample of veterans with OSA, we confirmed well-established predictors of daytime sleepiness (e.g., insufficient sleep, mood disorder diagnosis), but also we describe the less commonly recognized associations of medical comorbidities and the role of bed partners with reported daytime sleepiness. Age did not moderate the relationship between any of these variables and self-reported daytime sleepiness. In regard to insomnia symptoms, we verified well-known predictors of insomnia (e.g., age, sleep duration, mood disorder, and chronic pain diagnoses) and also identified (1) the negative association of Hispanic ethnicity with the ISI in OSA and (2) the positive association of higher educational attainment with the ISI in OSA. In addition, we found an interaction between age and sleep duration with the ISI. Our discussion focuses on these novel findings.

\section{Predictors of insomnia severity index in OSA}

Higher educational attainment was associated with greater insomnia symptoms. Our sleep clinic data diverge with population-based studies showing that individuals with higher educational attainment report significantly lower sleep-related complaints and insomnia [26]. It is unclear why higher education would have a detrimental relationship with insomnia symptoms among US veterans. Educational attainment may be a particularly weak individual-level SES measure for US veterans given their ability to afford college through their military benefits (e.g., GI bill) [27]. Thus, it is possible that the association between higher educational attainment and insomnia is mediated by other unmeasured variables such 
as financial strain or other psychosocial stressors [28]. These findings indicate that the relationship between education and insomnia symptoms among US veterans with OSA warrants further study.

Hispanic ethnicity was associated with lower ISI scores in veterans with OSA. Similar findings concerning insomnia complaints have been described in the National Health and Nutrition Examination Survey [9]. Mexican-Americans and other Hispanic individuals had a $40 \%$ and $30 \%$ lower likelihood of having difficulty falling asleep than whites, respectively. In addition, Mexican-Americans had 20\% lower likelihood of reporting difficulty maintaining sleep relative to whites. In our sleep clinic, the majority of Hispanic individuals (80\%) are of Puerto Rican and Cuban backgrounds, which may account for the association with the lower ISI scores [29]. As insomnia symptoms have been reported to vary within Hispanic subgroups [30], inquiring about Hispanic ethnicity may be an important contextual factor during insomnia symptom evaluation.

\section{Age and insomnia symptoms in OSA}

In accordance with community-based studies, younger age and shorter sleep duration were associated with greater insomnia symptoms in our sample with OSA [31]. The ISI queries insomnia symptoms as sleep "difficulties" and responses can vary considerably in two individuals with the similar perceived sleep onset latency or wake after sleep onset time. Thus, ISI scores may have a greater association with psychological distress than with objective sleep parameters [32]. In our sample, at any sleep duration, older veterans reported lower ISI scores than younger veterans (Fig. 1). These findings align with studies finding that older individuals are less likely to complain about sleep difficulties despite shifts towards lighter sleep architecture (greater stages N1 and N2, lower N3 and REM) and detrimental sleep continuity with aging (greater wake after sleep onset time) [33]. In the 2006 Behavioral Risk Factor Surveillance System, general sleep dissatisfaction was examined in over 150,000 US adults [34]. Difficulties with sleep were most often reported by young adults and sleep complaints generally declined with age. There are several potential explanations for this apparent paradox including that older individuals (1) may normalize long-standing sleep problems, (2) have less rigid social obligations/schedules allowing to nap during the day, or (3) possess stronger protective factors (e.g., better coping strategies or more robust social networks) than younger veterans.

In addition to the main effects of age on the ISI, an age-sleep duration interaction was observed. A 1-h change in sleep duration had a greater inverse effect on the ISI among older than younger veterans (Fig. 1). A possible explanation for this finding may be differential sleep duration and sleep expectations with aging. In contrast to epidemiological studies showing progressively shorter sleep duration with aging [33], we did not find any correlation between age and perceived sleep duration among veterans with OSA (Pearson correlation = $0.03 ; p=0.62$ ). For instance, the mean sleep duration was $5.4 \pm 1.5 \mathrm{~h}$ and $5.5 \pm 1.3 \mathrm{~h}$ for veterans $20-40$ years old and 60-80 years old, respectively. Given decreasing sleep duration needs with aging, this suggests that younger individuals may be more chronically sleepdeprived than older ones, making a 1-h extension in sleep duration less beneficial in improving insomnia complaints among younger than older veterans [33, 35]. In a sample of 
active US military personnel ( $n=725)$, Mysliwiec et al. found that nearly $42 \%$ of this young cohort reported sleeping $<5 \mathrm{~h}$ habitually [35]. Younger veterans may have a heavier burden of social responsibilities (e.g., working during the day, attending night school, child-rearing) cutting into their sleeping hours to a greater extent than among older individuals.

Additionally, expectations about sleep quality and duration among older individuals may be lower than those among younger veterans. This may be partially related to the beliefs that (1) older individuals do not need as much sleep as younger ones and (2) disturbed sleep is a normal part of the aging process [36]. Thus, a 1-h increase in sleep duration may have a more dramatic perceived improvement in insomnia complaints among older than younger individuals. These hypotheses need to be replicated in larger studies utilizing objective measures of sleep duration and stricter diagnostic insomnia criteria in OSA.

\section{Predictors of the Epworth sleepiness scale in OSA}

Surprisingly, a greater number of weighted medical comorbidities were associated with a lower level of daytime sleepiness. In a study exploring medical comorbidities utilizing the Charlson index to define clinical OSA clusters, Vavougios et al. reported one cluster (7\% of sample) with high mean CCI score but low levels of daytime sleepiness (ESS < 10) [37]. In another cluster analysis of OSA clinical phenotypes, Bailly etal. described one cluster (18\% of their sample) characterized by low ESS, many medical comorbidities, obesity, and high AHI [38]. Thus, it is possible that in our sample, similar OSA phenotypes may comprise a large portion of our sample and drive this association. One plausible explanation for the relationship between lower subjective sleepiness among those with greater medical comorbidities is that medical illnesses can represent a source of chronic stress and contribute to day-time hyperarousal [39]. However, this hypothesis warrants further exploration in future studies.

Relative to sleeping alone, the presence of a regular bed partner resulted in the report of higher level of daytime sleepiness. One possibility is an individual's increased awareness of dozing via the report of a regular witness. In particular, as most of our male veterans had female bed partners, gender differences in health risk concern and/or sensitivity to snoring may have contributed to this finding. Secondly, the bed partner's sleep routine, sleep-related movements, and/or untreated sleep disorders (e.g., snoring, insomnia) may negatively impact the individual's sleep quality. Interestingly, studies examining couples' nightly sleep covariation have shown that a male's total sleep time is predicted by the female partner's sleep duration but not vice versa [40]. Although the dyadic nature of sleep is recognized, there is little data on how it contributes to screening for sleep disorders or daytime functioning.

\section{Limitations}

Our study has additional limitations. First, an elevated ISI scores may not represent nocturnal insomnia symptoms in those with OSA. However, in a separate analysis of this cohort, using latent profile analysis of the ISI, we have showed that nearly $80 \%$ of our veterans have moderate to severe insomnia symptoms across all seven ISI items [41]. Only a small subset of veterans with OSA (9\%) had absent insomnia symptoms but elevated ISI scores. Another important limitation in the interpretation of these data is that the referral 
pattern to our sleep clinic may vary by age. Younger individuals who are more symptomatic from their OSA symptoms may be more likely to self-refer than older individuals with medical comorbidities referred by a physician. Our cohort consisted of a convenience sample of predominantly male veterans with OSA with a high prevalence of mental health comorbidities. Therefore, our results may not generalize other sleep apnea clinical samples. As previously mentioned, our insomnia and sleepiness symptoms relied on ISI and ESS responses alone and not objective measures of sleep (e.g., actigraphy, mean sleep latency test). Therefore, these insomnia or sleepiness symptoms may reflect other sleep disturbances (e.g., insufficient sleep) or that of untreated OSA. Finally, we did not adjust our analyses for use of medications on the night of diagnostic PSG. These limitations should be taken into consideration when interpreting our findings.

In conclusion, our data has important implications for the screening of comorbid insomnia symptoms among older veterans with OSA. Providers should consider evaluating insomnia symptoms assessing sleep parameters (e.g., sleep onset latency, wake after sleep onset time) instead of inquiring about sleep "difficulties." As many older individuals may sleep alone, focusing on objective parameters (e.g., BMI, neck circumference) may be a better OSA risk stratification strategy. Further studies need to be performed to develop personalized approaches towards querying insomnia symptoms among older US veteran samples with OSA.

\section{References}

1. Senaratna CV, Perret JL, Lodge CJ, Lowe AJ, Campbell BE, Matheson MC, Hamilton GS, Dharmage SC (2017) Prevalence of obstructive sleep apnea in the general population: a systematic review. Sleep Med Rev 34:70-81 [PubMed: 27568340]

2. Chowdhuri S, Patel P, Badr MS (2018) Apnea in older adults. Sleep Med Clin 13:21-37 [PubMed: 29412981]

3. Caldwell JA, Knapik JJ, Lieberman HR (2017) Trends and factors associated with insomnia and sleep apnea in all United States military service members from 2005 to 2014. J Sleep Res 26:665670 [PubMed: 28448689]

4. Ramos AR, Figueredo P, Shafazand S, Chediak AD, Abreu AR, Dib SI, Torre C, Wallace DM (2017) Obstructive sleep apnea phenotypes and markers of vascular disease: a review Front Neurol 8: 659 [PubMed: 29259576]

5. Onen F, Moreau T, Gooneratne NS, Petit C, Falissard B, Onen SH (2013) Limits of the Epworth sleepiness scale in older adults. Sleep Breath 17:343-350 [PubMed: 22467194]

6. Uhlig BL, Hagen K, Engstrom M, Stjern M, Gravdahl GB, Sand T (2018) The relationship between obstructive sleep apnea and insomnia: a population-based cross-sectional polysomnographic study. Sleep Med 54:126-133 [PubMed: 30554056]

7. Mysliwiec V, Gill J, Lee H, Baxter T, Pierce R, Barr TL, Krakow B, Roth BJ (2013) Sleep disorders in us military personnel: a high rate of comorbid insomnia and obstructive sleep apnea. Chest 144:549-557 [PubMed: 23681455]

8. Williams NJ, Grandne MA, Snipes A, Rogers A, Williams O, Airhihenbuwa C et al. (2015) Racial/ ethnic disparities in sleep health and health care: importance of the sociocultural context. Sleep Health 1:28-35 [PubMed: 26229976]

9. Grandner MA, Petrov ME, Rattanaumpawan P, Jackson N, Platt A, Patel NP (2013) Sleep symptoms, race/ethnicity, and socioeconomic position. J Clin Sleep Med 9:897-905 [PubMed: 23997702] 
10. Jackson M, Becerra BJ, Marmolejo C, Avina RM, Henley N, Becerra MB (2017) Prevalence and correlates of sleep apnea among us male veterans, 2005-2014. Prev Chronic Dis 14:E47 [PubMed: 28617665]

11. Kapur VK, Auckley DH, Chowdhuri S, Kuhlmann DC, Mehra R, Ramar K, Harrod CG (2017) Clinical practice guideline for diagnostic testing for adult obstructive sleep apnea: an American Academy of Sleep Medicine clinical practice guideline. J Clin Sleep Med 13:479-504 [PubMed: 28162150]

12. Collop NA, Anderson WM, Boehlecke B, Claman D, Goldberg R, Gottlieb DJ, Hudgel D, Sateia M, Schwab R (2007) Clinical guidelines for the use of unattended portable monitors in the diagnosis of obstructive sleep apnea in adult patients. Portable monitoring task force of the American Academy of Sleep Medicine. J Clin Sleep Med 3:737-747 [PubMed: 18198809]

13. American Academy of Sleep Medicine (2014) The AASM manual for the scoring of sleep and associated events: rules, terminology and technical specifications version 2.0. American Academy of Sleep Medicine, Westchester

14. D'Hoore W, Bouckaert A, Tilquin C (1996) Practical considerations on the use of the charlson comorbidity index with administrative data bases. J Clin Epidemiol 49:1429-1433 [PubMed: 8991959]

15. United States Census Bureau. American fact finder. https://factfinder.census.gov/faces/nav/jsf/ pages/index.xhtml. Accessed 10 Dec 2018

16. Bastien CH, Vallieres A, Morin CM (2001) Validation of the insomnia severity index as an outcome measure for insomnia research. Sleep Med 2:297-307 [PubMed: 11438246]

17. Johns MW (1991) A new method for measuring daytime sleepiness: the Epworth sleepiness scale. Sleep 14:540-545 [PubMed: 1798888]

18. Buysse DJ, Reynolds CF 3rd, Monk TH, Berman SR, Kupfer DJ (1989) The Pittsburgh sleep quality index: a new instrument for psychiatric practice and research. Psychiatry Res 28:193-213 [PubMed: 2748771]

19. Budhiraja R, Roth T, Hudgel DW, Budhiraja P, Drake CL (2011) Prevalence and polysomnographic correlates of insomnia comorbid with medical disorders. Sleep 34:859-867 [PubMed: 21731135]

20. Mysliwiec V, Matsangas P, Baxter T, McGraw L, Bothwell NE, Roth BJ (2014) Comorbid insomnia and obstructive sleep apnea in military personnel: correlation with polysomnographic variables. Mil Med 179:294-300 [PubMed: 24594464]

21. Ruiter ME, DeCoster J, Jacobs L, Lichstein KL (2010) Sleep disorders in African Americans and Caucasian Americans: a meta-analysis. Behav Sleep Med 8:246-259 [PubMed: 20924837]

22. Taylor DJ, Mallory LJ, Lichstein KL, Durrence HH, Riedel BW, Bush AJ (2007) Comorbidity of chronic insomnia with medical problems. Sleep 30:213-218 [PubMed: 17326547]

23. Chen E, Miller GE (2013) Socioeconomic status and health: mediating and moderating factors. Annu Rev Clin Psychol 9:723-749 [PubMed: 23245339]

24. Troxel WM (2010) It's more than sex: exploring the dyadic nature of sleep and implications for health. Psychosom Med 72:578-586 [PubMed: 20467000]

25. Hayes AF (2013) Introduction to mediation, moderation and conditional process: a regressionbased approach. The Guilford Press, New York

26. Grandner MA, Patel NP, Gerhman PR, Xie D, Sha D, Weaver T et al. (2010) Who gets the best sleep? Ethnic and socioeconomic factors related to sleep complaints. Sleep Med 11:470-478 [PubMed: 20388566]

27. Braveman P, Egerter S, Williams DR (2011) The social determinants of health: coming of age. Annu Rev Public Health 32:381-398 [PubMed: 21091195]

28. Hall M, Buysee DJ, Nofzinger EA, Reynolds CF, Thompson W, Mazumdar S et al. (2008) Financial strain is a significant correlate of sleep continuity disturbances in late-life. Biol Psychol 77:217-222 [PubMed: 18055094]

29. Wallace DM, Vargas SS, Schwartz SJ, Aloia MS, Shafazand S (2013) Determinants of continuous positive airway pressure adherence in a sleep clinic cohort of South Florida Hispanic veterans. Sleep Breath 17:351-363 [PubMed: 22528953]

30. Cespedes EM, Dudley KA, Sotres-Alvarez D, Zee PC, Daviglus ML, Shah NA, Talavera GA, Gallo LC, Mattei J, Qi Q, Ramos AR, Schneiderman N, Espinoza-Giacinto RA, Patel SR (2016) 
Joint associations of insomnia and sleep duration with prevalent diabetes: the Hispanic

Community Health Study/Study of Latinos (hchs/sol). J Diabetes 8:387-397 [PubMed: 25952169]

31. Grandner MA, Jackson NJ, Izci-Balserak B, Gallagher RA, Murray-Bachmann R, Williams NJ et al. (2015) Social and behavioral determinants of perceived insufficient sleep. Front Neurol 6: 112 [PubMed: 26097464]

32. Angst J, Gamma A, Clarke D, Ajdacic-Gross V, Rossler W, Regier D (2010) Subjective distress predicts treatment seeking for depression, bipolar, anxiety, panic, neurasthenia, and insomnia severity. Acta Psychiatr Scand 122:488-498 [PubMed: 20550521]

33. Ohayon MM, Carskadon MA, Guilleminault C, Vitiello MV (2004) Meta-analysis of quantitative sleep parameters from childhood to old age in healthy individuals: developing normative sleep values across the human lifespan. Sleep 27:1255-1273 [PubMed: 15586779]

34. Grandner MA, Martin JL, Patel NP, Jackson NJ, Gerhman PR, Pien G et al. (2012) Age and sleep disturbances among American men and women: data from the US Behavioral Risk Factor Surveillance System. Sleep 35:395-406 [PubMed: 22379246]

35. Mysliwiec V, McGraw L, Pierce R, Smith P, Trapp B, Roth BJ (2013) Sleep disorders and associated medical comorbidities in active duty military personnel. Sleep 36:167-174 [PubMed: 23372263]

36. Onen SH, Onen F (2018) Chronic medical conditions and sleep in the older adult. Sleep Med Clin 13:71-79 [PubMed: 29412985]

37. Vavougios GD, George DG, Pastaka C, Zarogiannis SG, Gourgoulianis KI (2016) Phenotypes of comorbidity in OSAS patients: combining categorical principal component analysis with cluster analysis. J Sleep Res 25:31-38 [PubMed: 26365653]

38. Bailly S, Destors M, Grillet Y, Richard P, Stach B, Vivodtzev I, Timsit JF, Lévy P, Tamisier R, Pépin JL, Scientific council and investigators of the French national sleep apnea registry (OSFP) (2016) Obstructive sleep apnea: a cluster analysis at time of diagnosis. PLoS One 11:e0157318

39. Adams RJ, Appleton SL, Vakulin A, Lang C, Martin SA, Taylor AW, McEvoy RD, Antic NA, Catcheside PG, Wittert GA (2016) Association of daytime sleepiness with obstructive sleep apnea and comorbidities varies by sleepiness definition in a population cohort of men. Respirology 21:1314-1321 [PubMed: 27312673]

40. Lee S, Martire LM, Damaske SA, Mogle JA, Zhaoyang R, Almeida DM, Buxton OM (2018) Covariation in couples' nightly sleep and gender differences. Sleep Health 4:201-208 [PubMed: 29555135]

41. Wallace DM, Wohlgemuth WK (2018) Predictors of insomnia severity index profiles among veterans with obstructive sleep apnea. Sleep 41:A221 


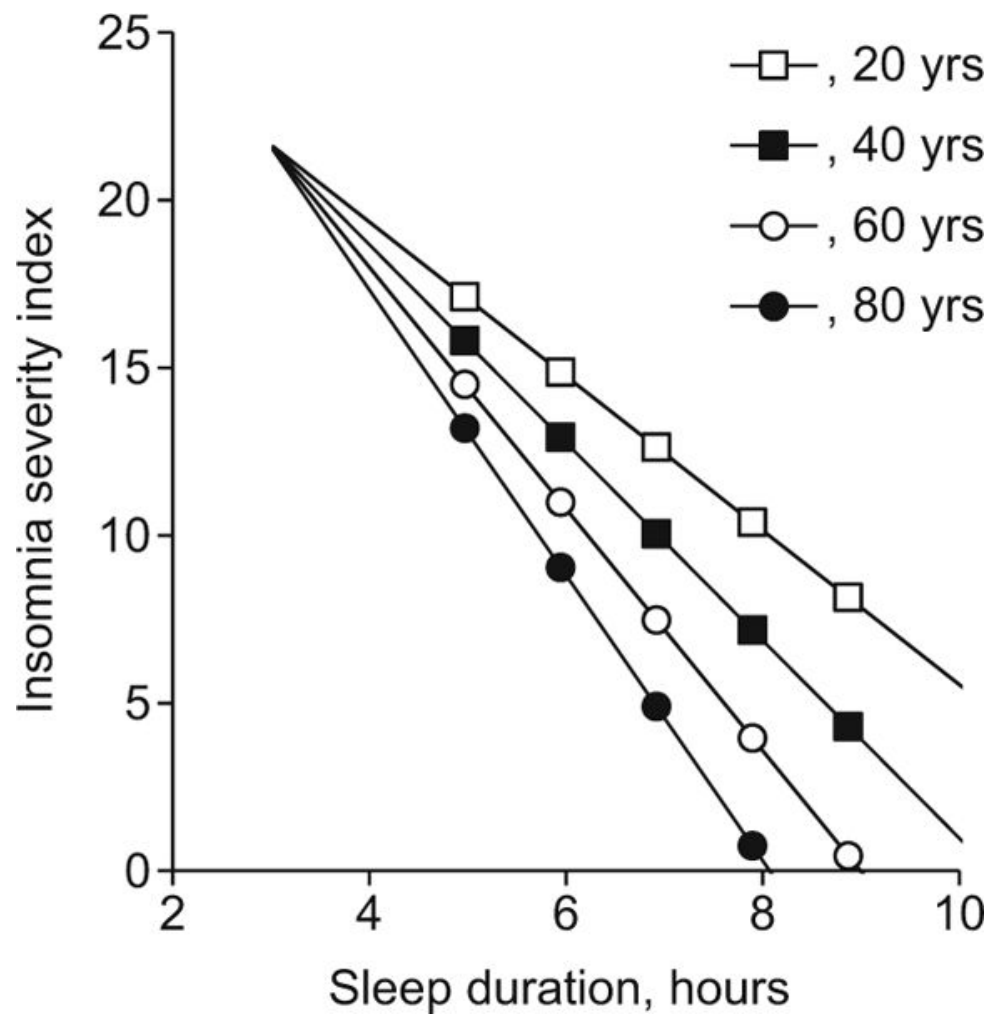

Fig. 1.

Regression lines for fully adjusted ISI model. Legend: A significant age-sleep duration interaction was noted for the insomnia severity index. Regression lines for fully adjusted models at 20-year age increments are shown. The simple slopes for the 20-, 40-, 60-, and 80year-old age groups were $-2.30,-2.95,-3.60$, and -4.26 , respectively. Each simple slope was significantly different than zero at $p<0.001$ 


\section{Table 1}

Descriptive and clinical characteristics of veterans with obstructive sleep apnea

\begin{tabular}{|c|c|}
\hline Characteristic & Total $(n=483)$ \\
\hline \multicolumn{2}{|l|}{ Demographics } \\
\hline Age (years) & $52 \pm 13$ \\
\hline Gender, $\%$ male & 93 \\
\hline Race, $\%$ black & 41 \\
\hline Ethnicity, \% Hispanic & 34 \\
\hline \multicolumn{2}{|l|}{ Anthropometrics } \\
\hline Body mass index $\left(\mathrm{m} / \mathrm{kg}^{2}\right)$ & $33 \pm 6$ \\
\hline \multicolumn{2}{|l|}{ Social variables } \\
\hline Marital status, $\%$ married & 52 \\
\hline Sleeping arrangement, $\%$ sleep alone & 30 \\
\hline Education, $\%$ high school or lower & 26 \\
\hline Median household income, $\mathrm{K}$ annually & $50 \pm 17$ \\
\hline \multicolumn{2}{|l|}{ Medical comorbidities } \\
\hline Hypertension & 63 \\
\hline Diabetes & 24 \\
\hline Cardiovascular disease & 18 \\
\hline Obstructive pulmonary disease & 14 \\
\hline Charlson comorbidity index & $1.3 \pm 1.7$ \\
\hline Chronic pain & 57 \\
\hline \multicolumn{2}{|l|}{ Psychiatric comorbidities } \\
\hline Mood disorder & 56 \\
\hline Post-traumatic stress disorder & 32 \\
\hline \multicolumn{2}{|l|}{ Sleep questionnaires } \\
\hline Insomnia severity index & $18 \pm 6$ \\
\hline Epworth sleepiness scale & $12 \pm 6$ \\
\hline \multicolumn{2}{|l|}{ Pittsburgh sleep quality index } \\
\hline Sleep onset latency (mins) & $41 \pm 38$ \\
\hline Sleep duration (h) & $5.4 \pm 1.3$ \\
\hline Time in bed (h) & $7.0 \pm 1.7$ \\
\hline \multicolumn{2}{|l|}{ Polysomnographic variables } \\
\hline PSG type, $\%$ in-lab & 76 \\
\hline Oxygen saturation nadir & $85 \pm 7$ \\
\hline AHI (events/h of sleep) & $36 \pm 28$ \\
\hline
\end{tabular}

Data presented as mean $\pm \mathrm{SD}$ or frequency (\%). $P S G$, polysomnography; $A H I$, apnea-hypopnea index 


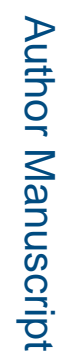

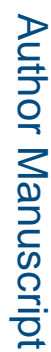

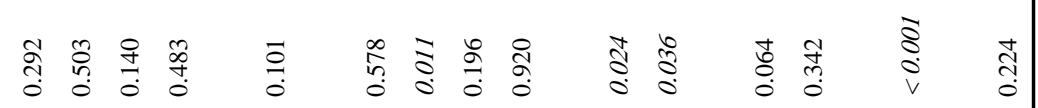
迎 రి

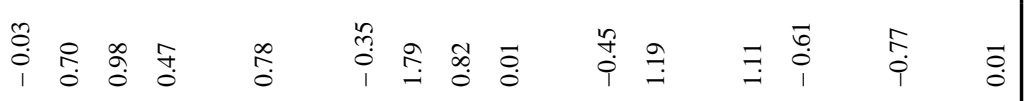




\section{ᄅ}

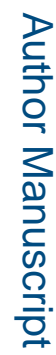

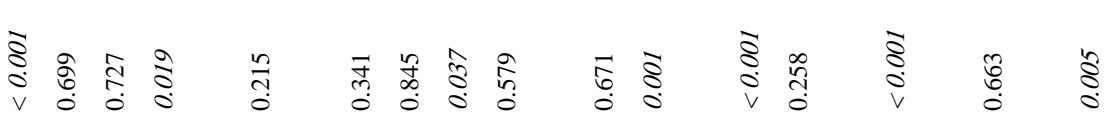

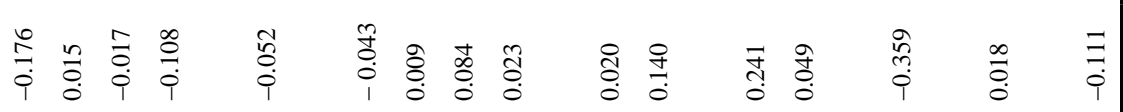

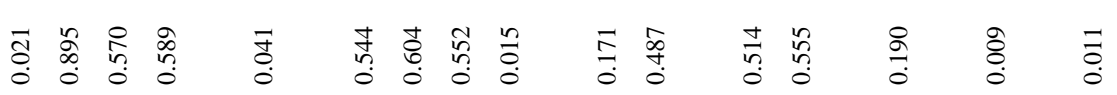

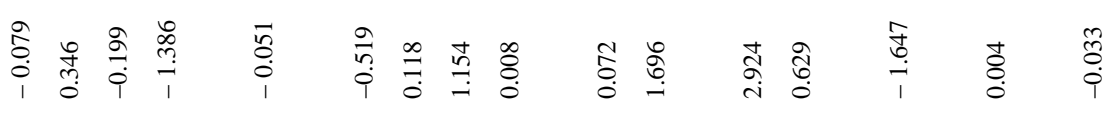

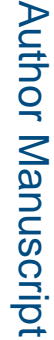

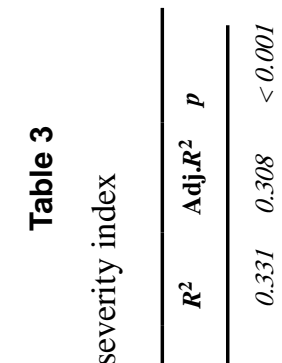

을
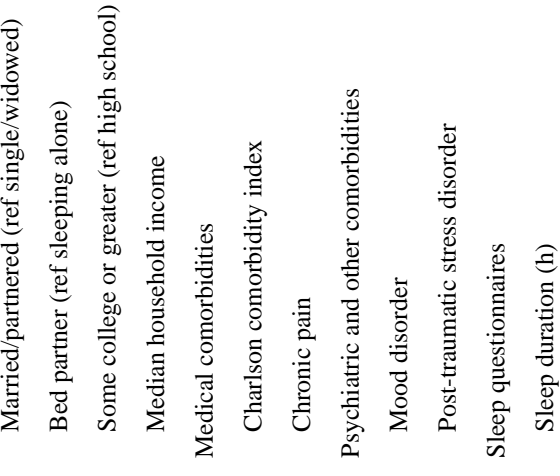